\title{
Plasticity in pentacene thin films
}

\author{
L. F. DRUMMY*,P. K. MISKA $\ddagger$ D. C. MARTIN $\$, \dagger$ \\ Department of Materials Science and Engineering, ${ }^{\dagger}$ Department of Biomedical Engineering \\ and the Macromolecular Science and Engineering Center, University of Michigan, \\ 2022 H. H. Dow Building, Ann Arbor, MI 48109-2136, USA \\ E-mail:milty@umich.edu
}

We have investigated the structure, defects and plasticity of thermally evaporated thin films of the organic molecular semiconductor pentacene using X-ray Diffraction (XRD), Optical microscopy (OM), Transmission Electron Microscopy (TEM), Electron Diffraction (ED), and High Resolution Electron Microscopy (HREM). Using XRD the degree of (001) texturing present in the as-grown films was characterized. The nature of pentacene plasticity and deformation-induced molecular alignment was investigated using rubbing and scratching techniques, as well as nanoindentation. Rubbing of the bulk powder produced thin oriented films, and a deformation length scale dependence was seen. Under stress pentacene crystals initially fail by cracking, until they reach a critical size of about one micron, when they tend to plastically deform into thin sheets. Alignment of thermally evaporated films was achieved under a controlled load scratch, and the degree of molecular orientation inside the scratched region was directly imaged using HREM. Finally, using nanoindentation we measured pentacene's plastic hardness to be $0.25 \mathrm{GPa}$ at a loading rate $0.05 \mathrm{mN} / \mathrm{s}$. A loading rate dependence of the hardness and stiffness was measured, with thin films behaving harder and stiffer at faster indentation rates.

(c) 2004 Kluwer Academic Publishers

\section{Introduction}

Pentacene is a crystalline organic molecular material currently under investigation for use as the active layer in all-organic flexible electronic devices [1-3]. Thin films are commonly produced by thermal evaporation onto various crystalline or amorphous substrates. Clearly the density and distribution of charge carrier trapping sites, such as dislocations and grain boundaries, will play a key role in determining film transport properties [4]. The mechanical properties of pentacene, and many other molecular crystals in general, are not well known. For pentacene and materials like it to be developed and integrated into useful devices, a greater understanding of their mechanical behavior in the thin film form must be obtained.

The deformation mechanisms of molecular crystals combine characteristics of metals, liquid crystals and polymers. They tend to be soft like polymers, and their anisotropic molecular conformation, low molecular weight, weak inter-molecular interactions and lack of physical entanglements allow for significant molecular rotations-like in liquid crystals. The high degree of long range order present in thin films and single crystals also allows for the presence of well-defined lattice defects. Defect motion can thus become mechanism for material reorganization under stress-like in metals. Models by Peterson [5] and Young [6] have explained yielding in semicrystalline polymers in terms of the nucleation and thermal activation of dislocations with Burgers vectors parallel to the polymer chain direction in crystallites. Due to the high degree of crystallinity in molecular crystalline thin films, the deformation of these materials may be mediated by similar defects.

The degree of ordering present in thin films of organics varies considerably depending on the particular material and the processing steps used. In some cases, uniformly disordered structures are ideal for obtaining the best properties, such as in doped conducting polymers or light-emitting polymers. In these materials an isotropic distribution of dopant and polymer material is usually desired, and crystallization of the polymer disrupts this isotropy [7]. In other cases, such as molecular crystals for optoelectronic applications like pentacene, a high degree of order is necessary to achieve good transport properties. These materials are intrinsic semiconductors that transport carriers through $\pi-\pi$ interactions, and these interactions are maximized in the single crystal form.

Several methods have been used to align organic thin films with the goal of producing single crystal texture.

\footnotetext{
*Present address: Air Force Research Laboratory, 2941 Hobson Way, Bldg. 654, WPAFB, OH 45433, USA.

$¥$ Present address: Department of Polymer Science and Engineering, University of Massachusetts, Amherst, 120 Governors Drive, Amherst, MA 01003-4530, USA.

$\S$ Author to whom all correspondence should be addressed.
} 
These techniques include the Langmuir-Blodgett technique [8], epitaxy [9, 10], growth onto rubbed [11] or stepped [12] substrates, and stress-induced alignment. We will study the latter in this paper, by rubbing and scratching thermally evaporated pentacene thin films. Deposition of liquid crystals onto rubbed polymeric alignment layers is a common practice used commercially to orient liquid crystals for device applications. Geary et al. have modeled this alignment process as resulting from the shear stress directly under the rubbing cloth [13]. Alignment of molecular crystalline thin films has been achieved by Ehara et al., who investigated the optical polarization of glycine films before and after rubbing [14]. Chen et al. used electron diffraction to characterize the extent of alignment in rubbed films of $p$-sexithiophene, $p$-sexiphenyl, copper phthalocyanine and pentacene [15].

We have applied rubbing and scratching techniques to align pentacene films and characterized the extent of alignment. Most techniques used to measure orientation such as X-ray diffraction and optical absorption methods average over relatively large sample areas. The overall film alignment can be measured with these methods, but the details of the structure at the molecular level are averaged out. Using HREM, we are able to directly image length scales from the molecular repeat spacings of $0.5 \mathrm{~nm}$, to nanocrystalline domains $10-50 \mathrm{~nm}$ in size, to over one square micron of aligned material-all in one image. With this kind of detailed information we can not only measure the overall orientation of the films, we can also see the individual lattice defects such as dislocations and grain boundaries that accommodate local misorientation. It is also possible to measure spatial distributions of crystalline domain orientation and lattice defects, which cannot be done with large-area measurement techniques.

We have used nanoindentation to quantify the mechanical response of pentacene thin films as a function strain and strain rate. Nanoindentation has been useful in determining the hardness and modulus of bulk polymers and thin films [16]. The viscoelastic properties of soft materials can also be probed with very good spatial and temporal sensitivity. Creep and strain rate dependence have been seen in nanoindentation of polymers [17]. Considerable insights about the deformation characteristics of the material can be gained by imaging the plastically deformed zone after indentation [18].

\section{Experimental}

Pentacene powder as received from Aldrich was sublimed in a glass tube approximately two centimeters in diameter held under a vacuum of $\sim 10^{-4}$ torr. A flame heat source was held at the end of the tube containing the pentacene powder, and this created a temperature gradient along the tube. The substrates used were amorphous carbon coated mica and uncoated mica placed approximately one to two centimeters from the source pentacene powder.

A Rigaku fixed source with $\mathrm{Cu} \mathrm{K}_{\alpha}$ radiation and a Bruker 2D wire detector was used for X-ray diffraction on textured thin films. Optical microscopy was done using a Nikon Optiphot with crossed polarizers. SEM was done with a Philips XL30 FEG operating at $5 \mathrm{kV}$, and TEM was done on a JEOL 4000 EX operating under low dose conditions [19] at $400 \mathrm{kV}$. The total end point dose for destruction of crystallinity of pentacene at $400 \mathrm{kV}$ is $0.25 \pm 0.05 \mathrm{C} / \mathrm{cm}^{2}$. All electron diffraction patterns were taken at a dose of $10^{-4}$ to $10^{-3} \mathrm{C} / \mathrm{cm}^{2}$. HREM micrographs were typically taken with a magnification of $60 \mathrm{kX}$, a screen current of $10-15 \mathrm{pA} / \mathrm{cm}^{2}$, and an exposure time of $1 \mathrm{~s}$. The dose of electrons $D$ received by the sample can be estimated by the equation:

$$
D=j M^{2} t
$$

where $j$ is the current density at the screen, $M$ is the magnification and $t$ is the exposure time. The imaging conditions used for HREM correspond to a dose $D$ received at the sample of $0.045 \pm 0.010 \mathrm{C} / \mathrm{cm}^{2}$, which is well below the critical dose of $0.25 \mathrm{C} / \mathrm{cm}^{2}$. A minimum dose system was used to control the microscope such that the sample was irradiated with a significant dose of electrons only during imaging.

Polycrystalline pentacene thin films were scratched on a Teledyne-Taber scratch tester with adjustable load and a 76 micron radius spherical diamond tip. The loads used to scratch were 0.1-1 grams. For TEM analysis, scratches were placed on pentacene films grown on amorphous carbon coated mica substrates. The amorphous carbon layer was approximately $20 \mathrm{~nm}$ in thickness. Many of the scratches significantly deformed and thinned the pentacene, however film structural integrity was maintained. Scratched pentacene films on the amorphous carbon support film were then floated off of the mica sheets onto deionized water for collection with copper TEM grids. A Nano Instruments Nano II was used to perform the nanoindentation tests. A Berkovitch tip was used, with maximum loads ranging from 0.2 to $10 \mathrm{mN}$ and loading rates from 0.015 to $0.7 \mathrm{mN} / \mathrm{s}$. A quartz standard sample was used to calibrate the instrument and calculate the tip area function.

\section{Results and discussion}

\subsection{As-grown film structure \\ 3.1.1. Crystal structure}

Pentacene single crystals have a triclinic structure $(\mathrm{P} \overline{1})$ with two molecules in the unit cell and lattice parameters $a=0.628 \mathrm{~nm}, b=0.771 \mathrm{~nm}, c=1.444 \mathrm{~nm}, \alpha=$ $76.75^{\circ}, \beta=88.01^{\circ}, \gamma=84.52^{\circ}$ and $\rho=1.365 \mathrm{~g} / \mathrm{cm}^{3}$ [20]. This crystal structure has been experimentally determined using X-ray diffraction from large $(\sim 1 \mathrm{~cm})$ crystals produced by slow solution growth under vacuum [20] and growth from the vapor in an inert gas atmosphere [21]. Like many aromatic molecules, pentacene packs in a herringboned arrangement in the $a-b$ plane. The $c$-axis is significantly longer than $a$ or $b$ and is assigned to the vector closest to the (001) normal. The (001) plane spacing, which is $1.41 \mathrm{~nm}$, is much larger than any other and becomes a signature spacing for the crystal. The molecules tilt away from the (001) normal toward the [1 $\overline{1} 0]$ direction. It has been presumed that 
polycrystalline films grow with the (001) planes parallel to the substrate, although the degree of texturing has not been measured in these films.

Thin films of triclinic bulk phase pentacene, well described by the above structure, can be produced by thermal evaporation of films greater than $100 \mathrm{~nm}$ in thickness onto substrates held at elevated temperature (50$100^{\circ} \mathrm{C}$ ) [22-25]. A unique "thin-film" phase has been found to grow in films of thickness generally less than $100 \mathrm{~nm}$ grown on substrates held at room temperature or colder, and it has an interlayer spacing ranging from 1.50 to $1.55 \mathrm{~nm}[4,22-25]$. The two processing parameters that most reproducibly cause a polymorphic structural transition in pentacene are film thickness and substrate temperature [23-25]. Variation of these two parameters can produce films with both the bulk phase and thin-film phase present. The thin-film phase has been found to grow in intimate contact with the substrate, and then at an apparently critical thickness the bulk phase begins to form [23]. Recently a new crystal structure has been found for thin film phase pentacene [26]. Crystals with an orthorhombic symmetry were characterized using high resolution electron microscopy and electron diffraction. The crystals had lattice parameters of $a=0.59 \pm 0.02 \mathrm{~nm}, b=0.74 \pm 0.02 \mathrm{~nm}$ and $c=3.00 \pm 0.08 \mathrm{~nm}$, with four molecules in the unit cell. In this structure, the molecules retain their herringboned packing in the $a-b$ plane, and they also herringbone along the $c$-axis. In this paper we study the deformation of the bulk triclinic phase of pentacene.

\subsubsection{Film morphology}

When thermally evaporated and condensed onto a substrate, pentacene commonly grows into textured polycrystalline films such that the (001) planes are parallel to the substrate across the entire film but the individual grains have random orientation in the plane. A transmission optical micrograph taken under crossed polars of a thermally evaporated pentacene film grown on mica is shown in Fig. 1. In the micrograph, a well-connected polycrystalline film can be seen. A two dimensional XRD pattern from a pentacene film on a glass substrate taken with the incident beam parallel to the film surface

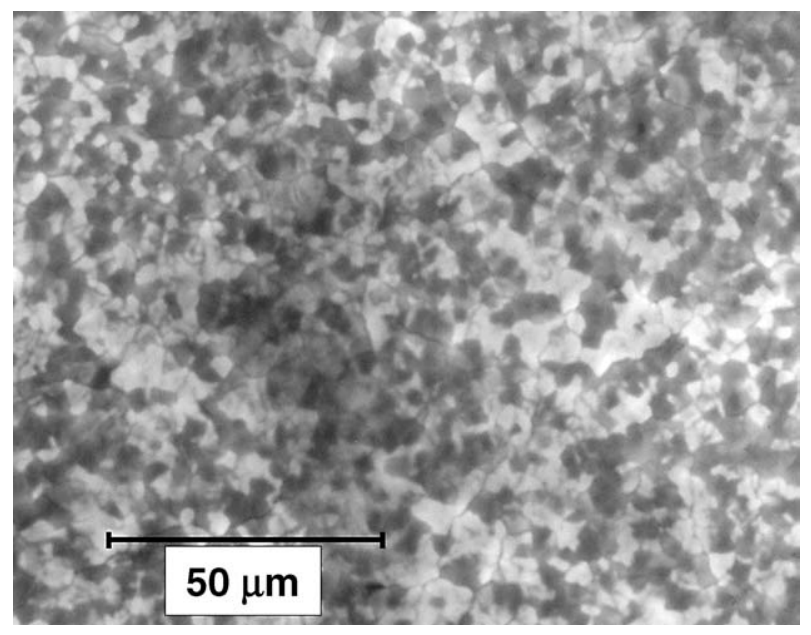

Figure 1 Optical micrograph of a polycrystalline pentacene thin film.

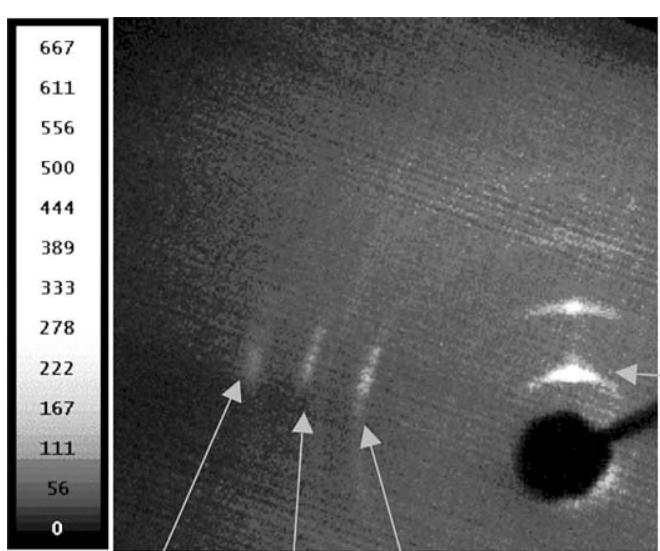

(123)

(022)

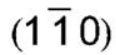

Figure 2 WAXS pattern of a textured pentacene film on a glass substrate taken with the incident beam parallel to the film surface.

is shown in Fig. 2. As can be seen in the intensity distribution of the (001) and (002) reflections, a large population of highly oriented crystals is present, along with a distribution of less oriented material as well. A planview bright-field TEM micrograph of a polycrystalline pentacene film on a thin amorphous carbon substrate is shown in Fig. 3, with electron diffraction patterns taken from two different single crystalline regions of the triclinic structure inset. The [111] orientation is the direction looking parallel to the long molecular axis of pentacene where the herringbone packing is most visible. The [112] orientation is close to the $(001)$ normal in the triclinic crystal structure and is thus a very common projection in plan-view from these textured films. In the TEM micrograph many (hk0) type grain boundaries and some holes between the grains can be seen. The dark bands in the crystal are diffraction patterns from bend contours. Under electron irradiation these bend contours move and interact with each other and defects in the crystal until they disappear when the crystallinity in the sample is destroyed. A region of sample that has received the critical dose of electrons and is totally amorphous with no bend contours can be seen running horizontally across the bottom region of the micrograph.

\subsection{Mechanical behavior}

\subsubsection{Rubbing of the bulk powder}

In previous work, pentacene microcrystals were mechanically sheared between glass slides to produce thin nanocrystalline films from which direct images of grain boundaries and dislocations were obtained with lattice resolution [27]. Here we have investigated the deformation characteristics of pentacene by rubbing of the bulk powder, controlled load scratching of thermally evaporated thin films, and nanoindentation. The bulk powder was rubbed on glass or amorphous carbon coated mica substrates using a glass slide or a razor blade. Fig. 4 shows a bright-field TEM micrograph of rubbed pentacene crystals on an amorphous carbon substrate, with the rubbing direction from the bottom of the image to 


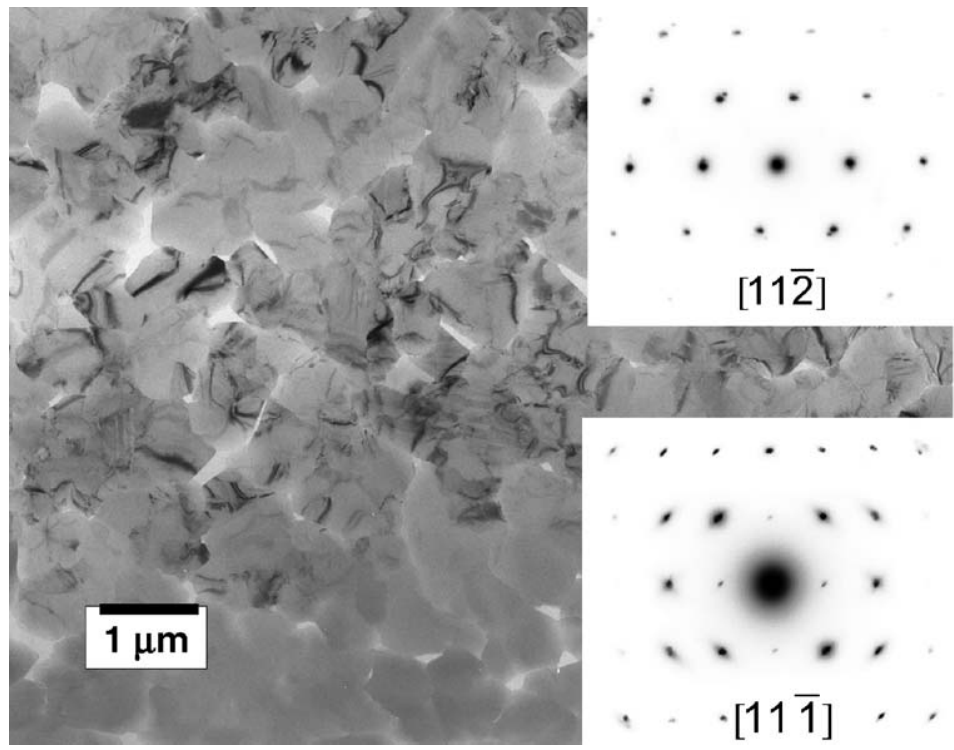

Figure 3 Plan-view TEM micrograph of a textured polycrystalline pentacene film with a grain size of $\sim 1 \mu \mathrm{m}$. Two electron diffraction patterns are inset, taken from two different single crystalline regions of the film in the [111] ] and [112] orientations.

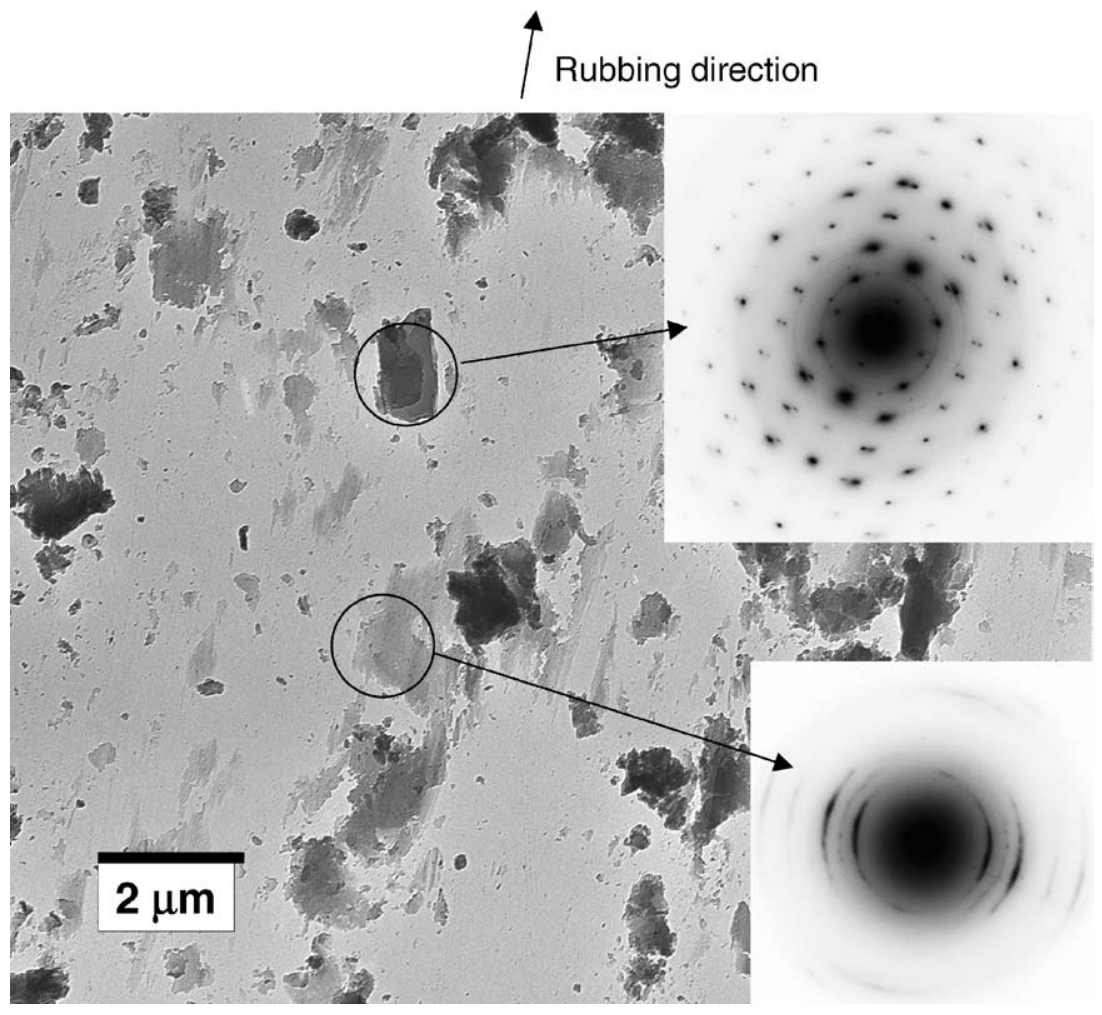

Figure 4 Bright-field TEM micrograph of pentacene crystals rubbed in one direction on an amorphous carbon substrate. The rubbing direction is from bottom to top. Electron diffraction patterns from two different regions are inset.

the top. The crystals were rubbed using a razor blade on the amorphous carbon coated mica, and the sample and carbon film was then floated off the mica onto deionized water for collection with copper TEM grids.

In the Fig. 4 two distinct morphologies can be seen. There are chunks of single crystals left behind, which are much smaller than the original bulk crystals and have not undergone significant plastic deformation. The electron diffraction pattern from one of these crystals (inset, top right) shows a single crystal structure in the [11]1] projection. There are also very thin regions of highly plastically deformed material which are $\sim 1 \mu \mathrm{m}$ in lateral size or less. The inset ED pattern (bottom right) shows that the thin plastically deformed sheets are aligned. These observations reveal that above a critical length scale of approximately one micron, fracture is the dominant mode of failure for pentacene crystals under stress. As crystals crack and reduce to a size of $\sim 1$ micron, defect-mediated plasticity then becomes the primary mode of deformation. This phenomenon can be qualitatively explained by the presence of surface defects or internal voids that have an intrinsic spacing near the critical length scale of approximately one micron. Cracks will presumably nucleate and propagate from such defects. When the crystals are smaller than this one micron defect spacing, other defects, such as 
dislocations, will mediate the deformation. The increasing tensile strength of diacetylene polymer fibers with decreasing diameter has been explained by a similar surface defect model [28].

With the use of HREM, we can measure the extent of alignment in the plastically deformed regions at the molecular length scale. Fig. 5a shows a HREM image of the rubbed film displayed at low magnification so that the rubbing direction can clearly be seen. This image is $1000 \mathrm{~nm}$ by $700 \mathrm{~nm}$ in size. When we zoom in to an image width of approximately $100 \mathrm{~nm}$ (Fig. 5c), we can view the $1.4 \mathrm{~nm}$ (001) lattice spacing, corresponding to the pentacene end-to-end molecular repeat spacing. There is significant local variation in the orientation of the (001) planes both between and even within a single crystalline domain. There is also evidence for dislocations within the deformed film. To measure the extent of alignment of the (001) planes, a Fast Fourier Transform (FFT) was taken from a $700 \mathrm{~nm} \times 700 \mathrm{~nm}$ region of the
HREM image (Fig. 5b). The azimuthal intensity profile measured from the FFT at a reciprocal space distance of $k=0.7 \mathrm{~nm}^{-1}$, corresponding to the pentacene (001) planes, is shown in Fig. 6. We measured an orientation factor $f=0.50$ for the (001) planes by fitting the following equation to the intensity data, where $I$ is the intensity measured at a given azimuthal angle $\phi$.

$$
\begin{aligned}
f & =\frac{3}{2}\left\langle\cos ^{2} \phi\right\rangle_{\mathrm{av}}-\frac{1}{2} \\
\left\langle\cos ^{2} \phi\right\rangle_{\mathrm{av}} & =\frac{\int_{0}^{\pi}\left(\cos ^{2} \phi\right)(I[\phi])(\sin \phi) d \phi}{\int_{0}^{\pi}(I[\phi])(\sin \phi) d \phi}
\end{aligned}
$$

An orientation factor of 1 indicates uniform alignment in the deformation direction, 0 indicates randomly oriented material, and -0.5 indicates the material is aligned in the direction normal to the deformation direction. These results, which characterize the nature of
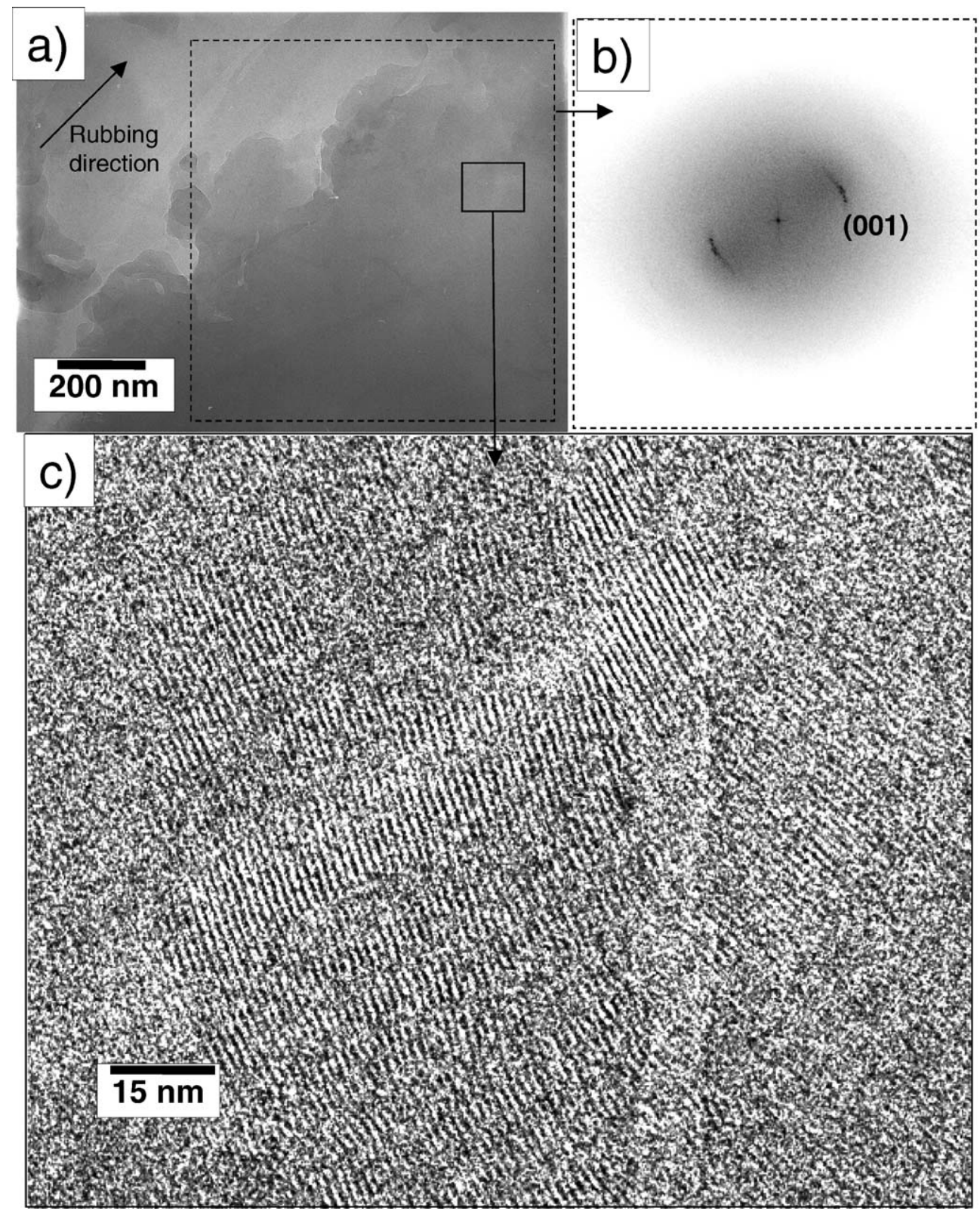

Figure 5 (a) HREM micrograph of a thin oriented pentacene sheet displayed at low magnification. The rubbing direction is indicated by the arrow. (b) Digital FFT from a $700 \mathrm{~nm}$ by $700 \mathrm{~nm}$ region of the image shows strong alignment of the $1.4 \mathrm{~nm}$ pentacene (001) planes perpendicular to the rubbing direction. (c) HREM micrograph of a small region of the deformed film with the $1.4 \mathrm{~nm}(001)$ fringes visible and nominally aligned perpendicular to the rubbing direction. 
(001) Plane Orientation

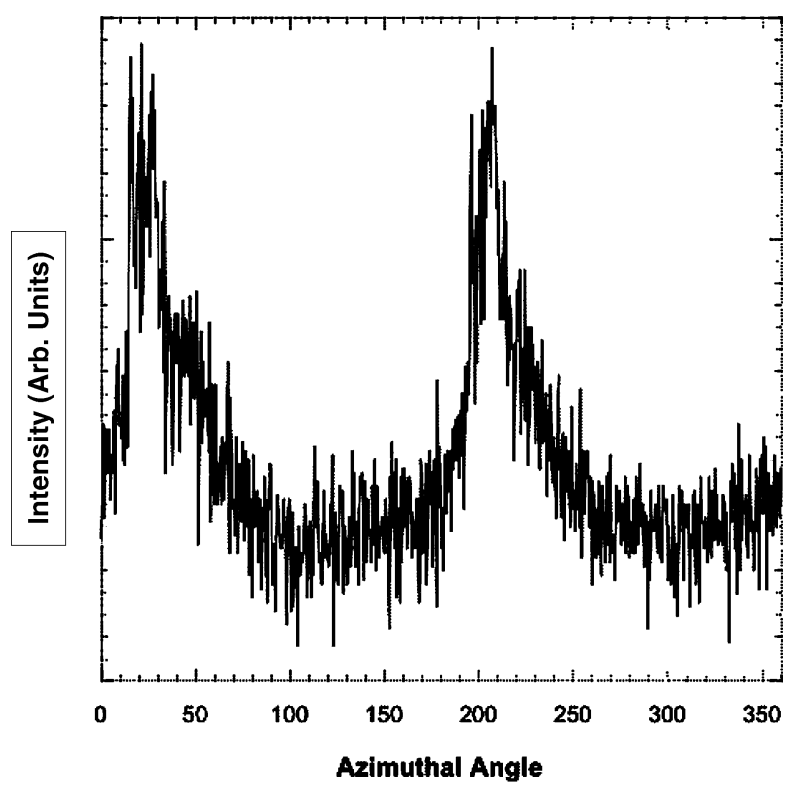

Figure 6 Azimuthal intensity profile of the FFT in Fig. 5b taken at a reciprocal space distance of $k=0.7 \mathrm{~nm}^{-1}(d=1 / k=1.4 \mathrm{~nm})$ corresponding to the (001) plane orientational distribution in the rubbed film.

pentacene deformation at many length scales, demonstrate that the material undergoes extensive reorganization under stress. The crystals before deformation are isotropically oriented, and after deformation they are aligned with the molecular long axis parallel to the substrate and having a preferred orientation in the rubbing direction.

\subsubsection{Nanoindentation of thermally evaporated films}

Nanoindentation was used to investigate the mechanical response of thermally evaporated pentacene thin films as a function of indentation depth. Fig. 7 shows loaddisplacement curves for several maximum loads and

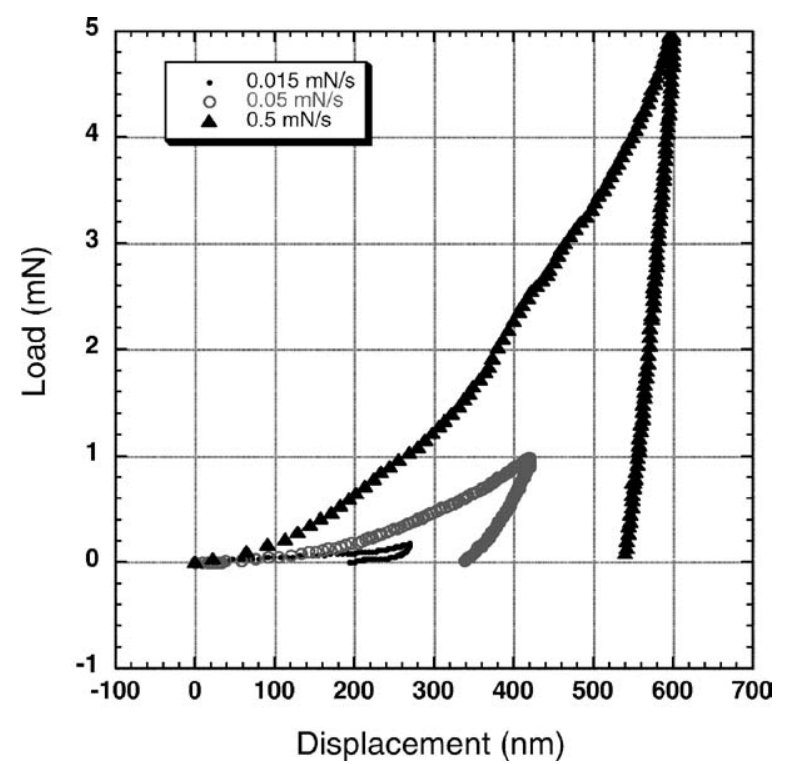

Figure 7 Nanoindentation load vs. displacement curves for several maximum loads and loading rates. loading rates. There is a significant amount of plastic deformation (low percentage of elastic recovery) evident in the curves even for very low applied loads (down to $0.2 \mathrm{mN}$ ). The apparent plastic hardness of the films can be calculated by dividing the applied load $P$ at a given displacement by the area $A$ of the resulting indent (calculated with knowledge of the tip area function).

$$
H=P / A
$$

We can see in Fig. 7 that as the loading rate increases, the films appear harder. A SEM image of a $7 \mathrm{mN}$ maximum load indent is shown in Fig. 8. Substantial material pile-up is seen at the edges of the indent. The size of the indent is on the order of the grain size of the material, however the grain boundaries do not appear to inhibit plastic flow. A plot of the plastic hardness vs. the loading rate is shown in Fig. 9. The value of the

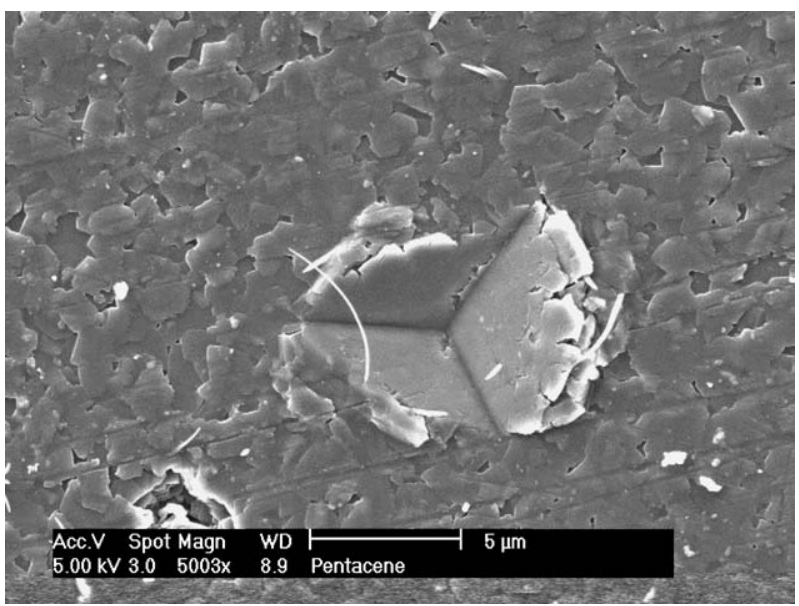

Figure 8 SEM micrograph of the plastically deformed region after a $7 \mathrm{mN}$ load indent.

\section{$\Longrightarrow$ Hardness (GPa)}



Figure 9 Elastic stiffness (squares) and plastic hardness (circles) vs. loading rate measured by nanoindentation of pentacene thermally evaporated films. 
hardness was calculated from the load vs. depth curves at an indentation depth of approximately $500 \mathrm{~nm}$, and for indents at low maximum loads which did not reach $500 \mathrm{~nm}$ depth, the hardness was calculated at the maximum depth for that curve. The error bars in the data represent one standard deviation of the hardness values collected for several indents at each loading rate. The line is a linear fit to the data, and an increasing trend in the plot can be seen over changes in the loading rate of nearly two orders of magnitude. The elastic stiffness was also calculated from the unloading portion of the curves. The stiffness is defined as:

$$
S=\mathrm{d} P / \mathrm{d} A
$$

and it can be seen in Figs 7 and 9 that there is a loading rate dependence of the elastic stiffness as well. The stiffness appears to increase rapidly at low loading rates $(<0.1 \mathrm{mN} / \mathrm{s})$ and then increases less rapidly at higher rates.

\subsubsection{Scratching of thermally evaporated films}

We have further investigated plasticity and deformation-induced alignment in pentacene by placing a controlled load scratch on thermally evaporated films. The as-grown films are textured with the (001) planes parallel to the substrate and the molecules standing nearly perpendicular to the substrate. Fig. 10 shows an optical micrograph taken under crossed polars of a scratched polycrystalline pentacene thin film lying on top of a copper grid. Birefringent single crystalline domains can be seen at a length scale of a few microns, and the scratch runs from the bottom left hand side of the image to the top right. A high degree of birefringence is seen in the scratched region indicating alignment. Optical microscopy is limited to

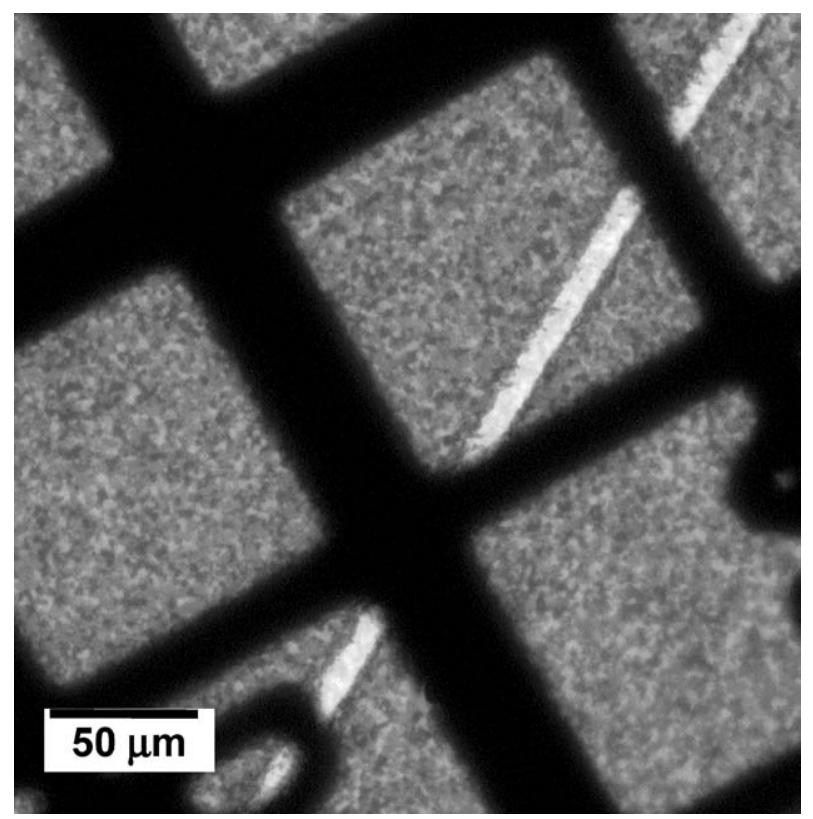

Figure 10 Optical micrograph taken under crossed-polars of a scratched pentacene thin film on a thin amorphous carbon substrate. The film is lying on top of a copper TEM grid.

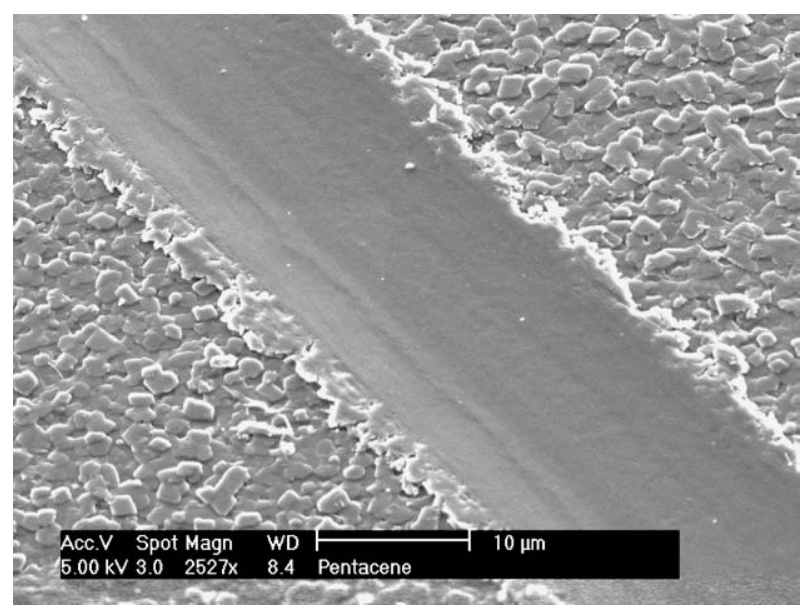

Figure 11 SEM image of a scratched pentacene thin film.

a resolution of approximately one micron, however. Using electron microscopy we can gain information about the deformed material at smaller length scales down to the molecular repeat spacings of the pentacene lattice.

A SEM image of the scratched region of a pentacene thin film is shown in Fig. 11. A clear change in the surface morphology is evident. The film is relatively rough at the crystal grain boundary length scale $(\sim 1 \mu \mathrm{m})$, and it is much smoother inside the scratch. Fig. 12 shows a bright-field TEM micrograph of the scratch. Outside the scratch there is a significant amount of diffraction contrast from bend contours indicating the high degree of crystallinity within each crystal grain. Mass-thickness contrast, arising from changes in sample density or

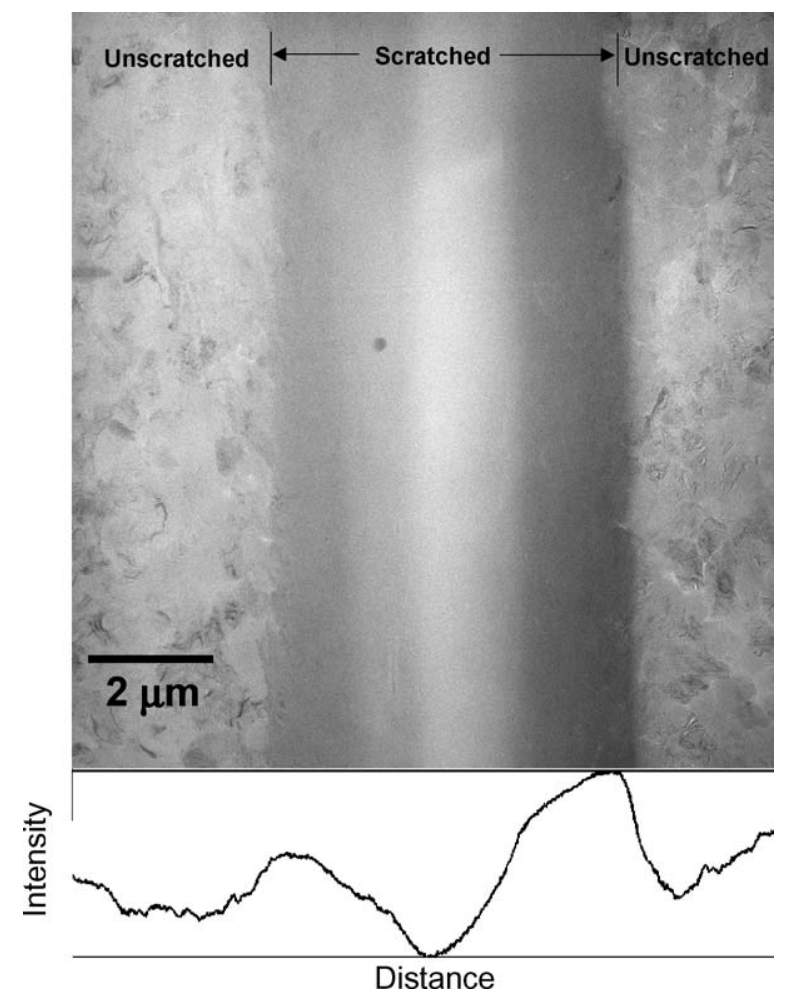

Figure 12 Bright field TEM micrograph of the scratch, which is vertical in the image. The integrated horizontal intensity line plot of the image is shown directly below. The value of the intensity in this plot is directly related to the mass multiplied by the thickness in the sample. 
thickness, can also be seen outside the scratched region near grain boundaries or small holes in the as-grown film. A remarkable difference in contrast can be seen between the scratched and unscratched regions. Inside the scratch the image contrast is extremely uniform, indicating uniform sample density on a $\sim 1 \mu \mathrm{m}$ length scale. These effects can be seen in an integrated line profile plot taken from the image, which is shown in Fig. 12 directly below the image. The plot is smoother in the scratched region. Also, significant sample thickening and material pileup at the edges of the scratch is evident in the darkening of the image there. However, though the material appears uniform on a $1 \mu \mathrm{m}$ length scale, we have found it is nanocrystalline inside the scratch with a grain size of approximately 20-50 nm.

Details about the deformation are obtained by HREM investigations within the scratch. Fig. 13 shows that there is high local orientation within nanocrystals of approximately $20-30 \mathrm{~nm}$ in size, but with considerable variation in the molecular orientation from region to region. Fig. 13a shows a HREM image from the center of the scratch with the $1.4 \mathrm{~nm}(001)$ fringes clearly visible and nominally aligned perpendicular to the scratch direction. The long axis of the molecules is therefore parallel to the scratch direction. Fig. 13b shows a dig- ital Fast Fourier Transform (FFT) taken from a $35 \mathrm{~nm}$ by $35 \mathrm{~nm}$ region of the image, which is approximately the size of one nanocrystalline domain. This particular crystal from which the FFT is taken is oriented in the [1]10] projection, giving evidence for a preferred contact plane of (110) type. A FFT taken from a much larger $300 \mathrm{~nm}$ by $300 \mathrm{~nm}$ region of the scratch (Fig. 13c) shows the overall degree of orientation across a wide region of the scratch. Many of the crystallites are oriented in the same [110] projection, as evident in the strong intensity of the off-axis reflections in Fig. 13c. These results demonstrate that this deformation process produces the beginnings of single crystal texture inside the scratch.

These thermally evaporated pentacene films have large crystals before alignment, and grain boundaries and holes between the grains. After scratching the holes are filled in, and the film is transformed from a microcrystalline state to a nanocrystalline state. The competition between grain size and overall film uniformity and its effect on charge transport has been investigated in thin films of copper phthalocyanine [29]. When grains are small the grain boundaries limit charge transport. However as the grain size is increased, and holes between the grains begin to form, these holes begin to

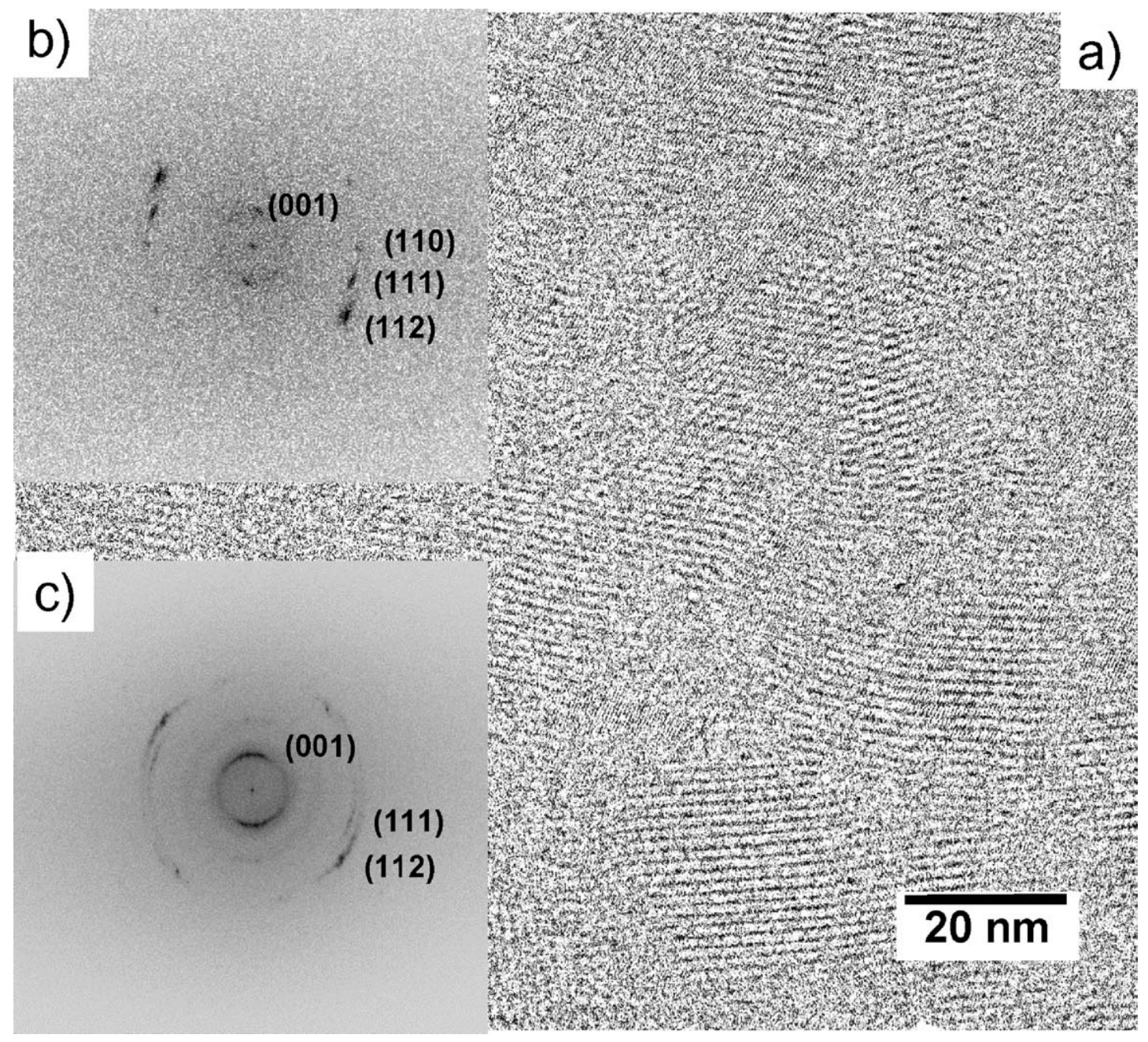

Figure 13 (a) HREM image from the center of the scratched region. (b) FFT from a $35 \mathrm{~nm}$ by $35 \mathrm{~nm}$ region of the image corresponding to the [1110] projection of the pentacene triclinic structure. (c) FFT taken from a $300 \mathrm{~nm}$ by $300 \mathrm{~nm}$ region of the scratch. A large degree of misorientation exists between individual crystal grains, however many crystals are in the same [110] projection, as indicated by the strong (111) and (112) reflections. 
degrade film electrical properties. This scratching method produces alignment of the films, and it also fills in the holes between grains with remarkable uniformity, as evident in the bright-field TEM micrograph (Fig. 12).

It may be possible to increase the grain size in the scratched region and reduce the defect density with annealing. This is a well-characterized process in metals, but its controllability has not been established in organic molecular crystals. The high vapor pressure of these materials makes it difficult to hold them at elevated temperatures for extended periods of time without causing film evaporatation. Annealing of the metal contacts in pentacene thin film transistors have shown

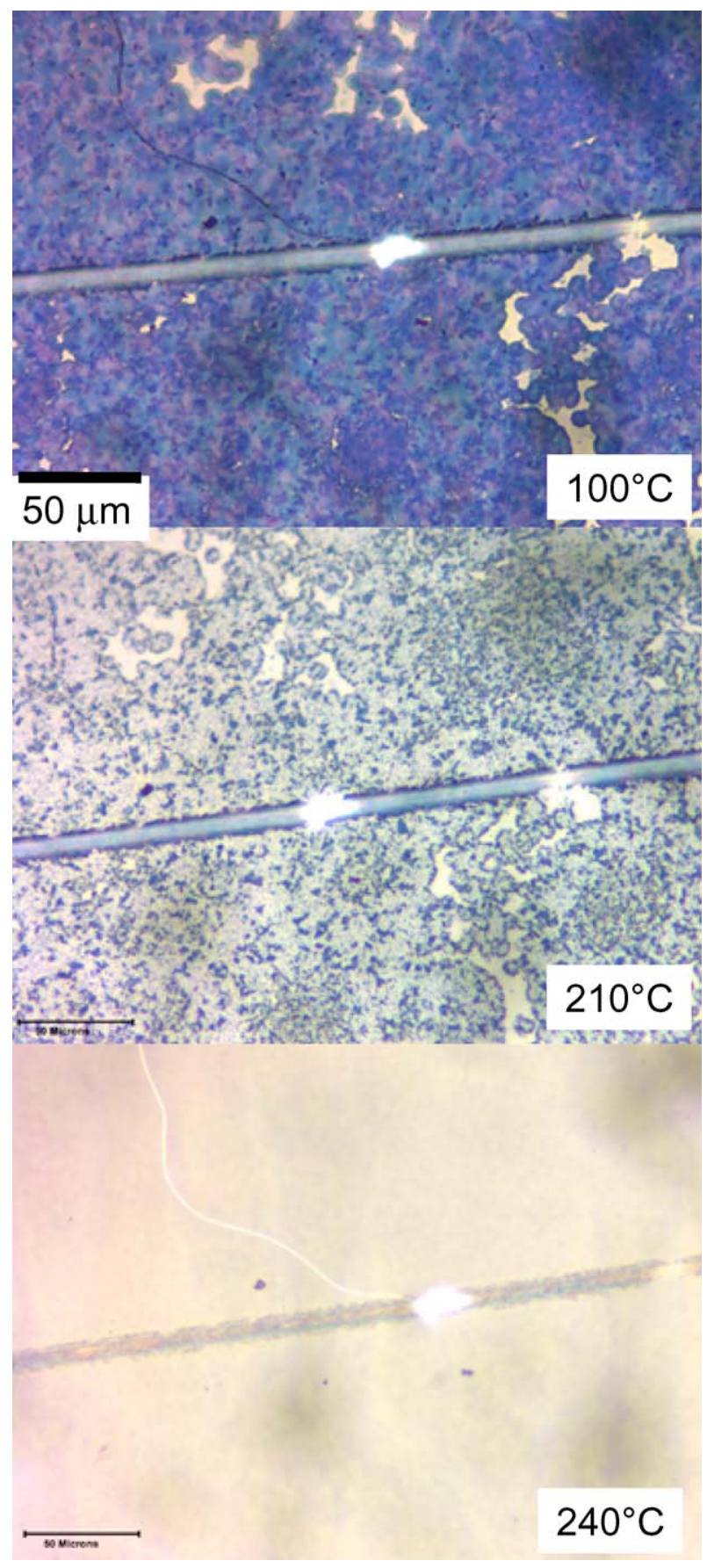

Figure 14 Optical micrographs of the same region of a scratched pentacene film on a carbon coated mica substrate at three different annealing temperatures. At $210^{\circ} \mathrm{C}$, much of the film has begun to sublime, however the scratched region remains intact. improvements in device properties [30], however no correlation with the annealing time and temperature has been made with pentacene film structure. We annealed a polycrystalline pentacene film that contained several controlled load scratches for one hour increments at constant temperature. No significant grain growth was seen in the films using polarized optical microscopy. At an annealing temperature of $210^{\circ} \mathrm{C}$, it was found that a significant amount of the film had evaporated. Three optical micrographs of the same region of a scratched film at three different annealing temperatures are shown in Fig. 14. While the much of the film has evaporated at $210^{\circ} \mathrm{C}$, the scratched region remains left behind. The change in vaporization behavior inside the scratched region may arise from the different orientation of the crystals there. Inside the scratch the molecules are oriented with their long axis, and $\pi$-electron orbitals, parallel to the substrate. In this state of orientation, the number of pentacene molecules per unit area at the free surface of the film is lower than in the (001) textured as-grown films. The change in vaporization behavior may also be caused by the change from the microcrystalline to the nanocrystalline state after deformation. The disordered state of the scratched film may kinetically inhibit evaporation. Similar to a "pick-up-sticks" game, the pentacene "sticks" are most easily picked up when they are highly ordered. We have shown that the film goes from uniformly dense microcrystals with a grain boundary spacing of $\sim 1 \mu \mathrm{m}$ before deformation, to a uniform film at the micron length scale with a grain boundary spacing of 20-50 $\mathrm{nm}$ after deformation.

\section{Conclusions}

We have characterized pentacene thin film structure before and after plastic deformation. Pentacene powder, consisting of single crystals $20-50 \mu \mathrm{m}$ in size oriented in all directions, was rubbed to produce thin sheets of aligned material. It was found that when pentacene crystals are large they tend to fail by cracking until they reach a critical size of $\sim 1 \mu \mathrm{m}$ and then plastically deform into thin oriented sheets. Thermally evaporated films were textured with a single crystal domain size of $\sim 1 \mu \mathrm{m}$ and the (001) planes parallel to the substrate before deformation. Under a controlled load scratch the material's grain size reduced to approximately $20 \mathrm{~nm}$ and the crystallites had a preferred orientation with $\mathrm{c}^{*}$ along the scratch direction and a contact plane of (110) type. Nanoindentation was used to measure the hardness of the films at a given indentation rate. A loading rate dependence was measured, as the films behaved harder and stiffer when indenting at faster rates. Pentacene films that contained several scratches were heated to the sublimation point, and at temperatures sufficient to volatilize the undeformed material the scratched region remained relatively intact.

The results presented here show that pentacene thin films are capable of significant amounts of plastic deformation and alignment under stress. Pentacene single crystals fail by fracture when they are larger than $1 \mu \mathrm{m}$, suggesting this is an optimum grain size for minimizing defect density while maintaining film structural integrity. The microcrystalline as-grown films, which 
have a fiber texture with $\mathrm{c}^{*}$ normal to the substrate, can be deformed by scratching to form a single crystal textured nanocrystalline film with $\mathrm{c}^{*}$ nominally aligned in the scratch direction and the (110) planes parallel to the substrate. It remains to be seen how this alignment process can best be used to maximize performance in a transistor device geometry. We have seen that scratched films have a high defect density at the nanometer length scale yet at the same time appear uniform at the micron length scale. It will be of interest to determine the effect of this length-scale dependant structural uniformity in the scratched films on their electrical transport properties.

\section{References}

1. C. D. DimitRAKOPOULOS and P. R. L. MALENFANT, Adv. Mat. 14 (2002) 99.

2. Y.-Y. LIN, D. J. GUNDLACH, S. F. NELSON and T. N. J AC K S O N, IEEE Trans. Electron Dev. 18 (1997) 87.

3. M. HALIK et al., Adv. Mat. 14 (2002) 1717.

4. J. G. LAQUINDANUM, H. E. KATZ, A. J. LOVINGER and A. DODAB ALAPUR, Chem. Mater. 8 (1996) 2542.

5. J. M. PETERSON, J. Appl. Phys. 37 (1966) 4047.

6. R. J. YOUNG, Phil. Mag. 30 (1974) 85.

7. J. MORGADO, F. CACIALli, J. GRUNER, N. C. GREENHAM and R. H. FRIEND, J. Appl. Phys. 85 (1999) 1784.

8. M. SUZUKI, A. FERENCZ, S. IIDA, V. ENKELMANN and G. WEGNER, Adv. Mat. 5 (1993) 359.

9. I. MOGGIO et al., Macromolecules 43 (2001) 7091.

10. H. YANAGi, S. DOUMI and T. SASAKI, J. Appl. Phys. 80 (1996) 4990.

11. M. L. SWIgGers et al., Appl. Phys. Lett. 79 (2001) 1300.

12. J. O. OSSÓ et al., Adv. Func. Mat. 12 (2002) 455.
13. J. M. GEARY, J. W. GOODBY, A. R. KMETZ and J. S. PATEL, J. Appl. Phys. 62 (1987) 4100.

14. T. EHARA, H. HIROSE, H. KOBAYASHI and M. KOT A I, Synth. Met. 109 (2000) 43.

15. X. L. CHEN, A. J. LOVINGER, Z. N. BAO and J. S A PJET A, Chem. Mat. 13 (2001) 1341.

16. A. V. KULKARNI and B. BHUSHAN, J. Mat. Res. 12 (1997) 2707.

17. M. OYEN-TIESMA, Y. A. TOIVOLA and R. F. COOK, MRS Proceedings 649 (2001) Q1.5.1.

18. C. J. BUCHKO, M. J. SLATTERY, K. M. KOZLOFF and D. C. MARTIN, J. Mat. Res. 15 (2000) 231.

19. D. C. MARTIN and E. L. THOMAS, Polymer 36 (1995) 1743.

20. D. HOLMES, S. KUMARASWAMY, A. J. MATZGER and K. P. C. VOLLHARDT, Chem. Eur. Journ. 5 (1999) 3399.

21. C. C. MAttheus et al., Acta. Cryst. C 57 (2001) 939.

22. D. J. GUNDLACH, Y.-Y. LIN, T. N. JACKSON, S. F. NELSON and D. G. SCHLOM, IEEE Electron Dev. Lett. 18 (1997) 87.

23. I. P. M. BOUCHOMS, W. A. SCHOONVELD, J. VRIJMOETH and T. M. KLAPWIJ K, Synth. Met. 104 (1999) 175.

24. C. D. Dimitrakopoulos, A. R. BROWN and A. POMP, J. Appl. Phys. 80 (1996) 2501.

25. T. JENTZCH, H. J. JUEPNER, K.-W. BRZEZINKA and A. LA U, Thin Solid Films 315 (1998) 273.

26. L. F. DRUMMY, P. MISKA and D. C. MARTIN, MRS Proceedings 734 (2003) A2.2.1.

27. L. F. DRUMMY, C. KÜBEL, D. LEE, A. WHITE and D. C. MARTIN, Adv. Mat. 14 (2002) 54.

28. C. GALiOTIS, R. T. READ, P. H. J. YEUNG and R. J Y OUNG, J. Polym. Sci. 22 (1984) 1589.

29. Z. N. BAO, A. J. LOVINGER and A. DODABALAPUR, Adv. Mat. 9 (1997) 42.

30. J. H. LEE et al., J. Kor. Phys. Soc. 38 (2001) 282.

Received 18 August 2003

and accepted 26 February 2004 